\title{
Fluctuation-response relations for nonequilibrium diffusions with memory
}

\author{
C. Maes, ${ }^{1}$ S. Safaverdi, ${ }^{1}$ P. Visco, ${ }^{2}$ and F. van Wijland ${ }^{2}$ \\ ${ }^{1}$ Instituut voor Theoretische Fysica, KU Leuven-Celestijnenlaan 200D, B-3001 Leuven, Belgium \\ ${ }^{2}$ Laboratoire Matière et Systèmes Complexes, CNRS UMR 7057, Université Paris Diderot, 10 rue Alice Domon et Léonie Duquet, \\ 75205 Paris cedex 13, France
}

(Received 16 November 2012; published 19 February 2013)

\begin{abstract}
Strong interaction with other particles or feedback from the medium on a Brownian particle entail memory effects in the effective dynamics. That motivates the extension of the fluctuation-dissipation theorem to nonequilibrium Langevin systems with memory. An important application is to the nonequilibrium modification of the Sutherland-Einstein relation between diffusion and mobility in the case of strong memory. Nonequilibrium corrections include the time correlation between the dynamical activity and the velocity of the particle, which in turn leads to information about the correlations between the driving force and the particle's displacement.
\end{abstract}

DOI: 10.1103/PhysRevE.87.022125

PACS number(s): 05.40.-a

\section{INTRODUCTION}

Path integrals are robust against small perturbations in the dynamics and hence make expansions easier, such as for the derivation of response relations. That has been systematically applied before for the extension of the fluctuation-dissipation theorem to nonequilibrium systems, at least in the Markov case [1-4]. The purpose of this paper is to further extend the nonequilibrium linear response theory to dynamics with memory, which is physically often more appropriate, and which has been so far considered only in a "weak" nonMarkovian case, where memory decreases exponentially fast $[5,6]$. Also, here the very name fluctuation-dissipation relation needs to be revised and perhaps altered, as the response obtains correlations both with the excess entropy flux (which is responsible for the standard relation with dissipation) and with the time-symmetric part of the action, which has been called the frenetic contribution as it ultimately relates to the dynamical activity in the process [3]. For more practical purposes, it is the latter frenetic contribution where the steady nonequilibrium forcing appears and, hence, fluctuation-response relations can yield information about that forcing. Applications such as to the mobility of particles in living cells are in progress [7], but in this paper we concentrate on the general framework, numerical exploration, and some technical details.

An important ingredient in this work is the presence of memory in the equations of motion of a colloidal particle. The origin of memory is diverse but it is always related to coupling with other particles and/or with the environment. In the case of dense colloidal suspensions, the reduced dynamics of a single particle certainly contains memory by integrating out the other particles. The theoretical study of the relation between its diffusion and its mobility is therefore advanced by the analysis of generalized Langevin equations (GLE). The latter also appear from other reduction and projection schemes as generally treated via Zwanzig-Mori techniques $[8,9]$. There, temporal scale separation or micro-macro transfer are the important considerations, but also intrinsic properties of the medium can contribute memory effects. For the latter, we have in mind viscoelastic media which react back on active particles from their previous history. These are of special interest for mesoscopic processes in tissues or membranes within living organisms which are known to respond more easily to external loads.

Here, we do not concentrate on the specific interactions or mechanism that have created the memory effects, but we start from driven GLE for which we assume a structure that is relevant for a large number of cases of suspensions under the influence of external forces. See [10] for a more recent microscopic-based derivation of GLE in the context of polymer physics. The most important element in our modeling scheme is the principle of local detailed balance. It derives from the underlying microreversibility, which gives a strong connection between entropy flux and time-reversal breaking [11]. As we will see in the next section, application of local detailed balance leads to the so-called Einstein relation, also called second fluctuation-dissipation relation, between friction and noise in the GLE [12], even when modeling nonequilibrium situations. We do not, however, pay special attention to the choice of driving, but we are interested here in general features and structures of the response. For a specific application of these general methods, we refer to the recent work [7] on reconstructing the active forces from quantitative information on the violation of the fluctuation-dissipation theorem. We believe, however, that many more applications are waiting, in fact in all these cases where one can measure deviations from the standard Kubo theory $[12,13]$.

The study of response in nonequilibrium suspensions is of course not new (see, e.g., [14-17] for applications to sheared media). In that respect, the present contribution starts from GLE and investigates what are the general structures that determine the linear response. In particular, the work of $[18,19]$ addresses very similar questions and uses MartinSiggia-Rose field theory to obtain a fluctuation-response theory. We concentrate on Sutherland-Einstein relations and we emphasize the structure in terms of entropic versus frenetic contributions, making contact with the Markov formulation in [1].

Close to this work are also the results relating energy dissipation to the difference of the response and velocity correlation functions $[4,20]$ also for GLE. Here, we emphasize, however, the modified Sutherland-Einstein relation connecting diffusion and mobility. 
In the next section, the setup is considered for generalized Langevin systems with Gaussian noise. They are driven away from equilibrium by nonconservative forces. We derive the linear response relations in Sec. III. These results are ready to be applied in a relation between diffusion and mobility for colloidal particles in nonequilibrium viscoelastic media. Section IIIE contains the simulation results for exploring the modified Sutherland-Einstein relation and adds visual information on the behavior of the various terms in the modified relation. The main result of the paper is the extension of the work in [1] to include (even strong) memory effects and to be explicit also about the relevance of the correlations with dynamical activity and with the forcing.

\section{THEORY}

\section{A. Setup}

Consider the Langevin equation for the position $x_{t}$ and the velocity $v_{t}$ of a (mass 1 ) particle in a medium at uniform temperature:

$$
\begin{aligned}
& \frac{d x_{t}}{d t}=v_{t}, \\
& \frac{d v_{t}}{d t}=-\int d s \gamma(t-s) v_{s}+F_{t}\left(x_{t}\right)+\sqrt{\frac{2}{\beta}} \eta_{t}+h_{t} .
\end{aligned}
$$

To lighten the notation, we shall consider that, unless otherwise specified, integral bounds are understood to range from $-\infty$ to $\infty$. We take the memory kernel $\gamma(t) \geqslant 0$ to be causal: $\gamma(t)=0$ for $t<0$. The Markov case with friction coefficient $\gamma>0$ is recovered whenever $\gamma(|t|)=2 \gamma \delta(t)$ is proportional to the Dirac delta function, which can be achieved for example from $\gamma(t)=\gamma \alpha \exp (-\alpha t) \Theta(t)$ in the $\alpha \rightarrow \infty$ limit, with $\Theta(t)$ the Heaviside step function. The $F_{t}$ is the forcing, possibly time dependent and nonconservative. It can include effective randomness beyond the Gaussian noise $\eta_{t}$ as, e.g., in [7]. The parameter $\beta$ is the inverse temperature of the environment, which we have taken in front of the noise $\eta_{t}$. The force $\eta_{t}$ is a stationary Gaussian noise process with zero mean. We wait to describe its time correlations [see formula (8)]. The last term $h_{t}=f_{t} \Theta(t)$ is a time-dependent (small) perturbation; we will linearly expand around $f_{t}=0$. For simplicity, we use a onedimensional notation, also in what follows, but the extension to other geometries or dimensions, sometimes essential for nonequilibrium effects, is straightforward. We will not use a Fokker-Planck description in what follows (but we use path integrals); actually, the relation between generalized Langevin and generalized Fokker-Planck equations in the presence of position-dependent forces is not entirely clear (see [21,22] for what we do know).

For path-space integration, we need some further notation. Easiest is to take doubly infinite paths $\omega=\left(x_{s}, v_{s},-\infty<\right.$ $s<+\infty)$. The price to pay is that some expressions (integrals) become rather formal. We refer to [23] for a more detailed reference. Feasible alternatives or complements to path integration to derive fluctuation-response relations for non-Markovian processes are known as Furutsu-Novikov theorems (see, e.g., [20,24-26]).

Because the noise $\eta_{t}$ is a stationary Gaussian process, the path-space measure is completely determined by the symmetric kernel $\Gamma(t)$ for which

$$
\int d s \Gamma(t-s)\left\langle\eta_{s} \eta_{r}\right\rangle=\delta(t-r)
$$

The weight of a path $\omega$ is then proportional to

$$
\mathcal{P}_{h}(\omega) \propto \exp -\frac{\beta}{4} \int d s \int d r \Gamma(r-s) \eta_{s} \eta_{r}
$$

with

$$
\eta_{s}=\dot{v}_{s}+\int d u \gamma(s-u) v_{u}-F_{s}\left(x_{s}\right)-h_{s} .
$$

Compared with the unperturbed dynamics $\left(h_{t} \equiv 0\right)$ we have

$$
\mathcal{P}_{h}(\omega)=\mathcal{P}_{0}(\omega) e^{-\mathcal{A}_{h}(\omega)}
$$

with action

$$
\begin{aligned}
\mathcal{A}_{h}(\omega)= & -\frac{\beta}{2} \int d s \int d r \Gamma(r-s) h_{s} \dot{v}_{r} \\
& -\frac{\beta}{2} \int d s \int d r \int d u \Gamma(r-s) \gamma(r-u) v_{u} h_{s} \\
& +\frac{\beta}{2} \int d s \int d r \Gamma(r-s) h_{s} F_{r}\left(x_{r}\right)+O\left(h^{2}\right)
\end{aligned}
$$

to first order in the perturbation $h_{t}$. For stochastic integration and path integrals in the non-Markovian case, see also [23] and the more recent [27] with additional references. Equations (4) and (5) define our dynamical ensemble for the path-space distribution with respect to the unperturbed dynamics (1).

Finally, a word about initial and boundary conditions, also important for the terminology. As should be clear from the start, the dynamics (1) is not microscopic and its content depends on chosen levels of description, including spatiotemporal scales. In the discussion of diffusion, one assumes no spatial confinement over the relevant time scales. The behavior is then transient concerning the position degrees of freedom, but beyond the inertial regime the velocities relax and become Maxwellian. In that regime, overdamped approximations can be valid, which can be formally obtained in what follows by setting $\dot{v}=0$. For the general question of linear response, we also have in mind the case where the force $F_{t}=F+K_{t}$ contains a time-independent conservative force $F=-\nabla U$ from a normalizable and confining potential $U$. Under such a confinement and for time-independent forcing $K_{t}=K$, it is then assumed that there is a unique and smooth stationary density $\rho(x, v)$ to which all initial data converge. We speak of an equilibrium dynamics when $K_{t}=0$ (only confining potential). The case of free diffusion $F_{t}=0$ is, however, not strictly equilibrium as it need not be stationary even in the velocity degrees of freedom. For example, for free diffusion the Sutherland-Einstein relation will only be recovered when the initial data are also randomly chosen from a Maxwellian. In the formalism following, the important property of full equilibrium will be stationarity combined with time-reversal invariance. In fact, to the dynamics (1) must still be added a relation between the noise correlations and the memory kernel so as to ensure, for example, that for $F_{t}=0$ the velocities become Maxwellian. The next section takes this to a more general discussion by formulating the condition of local detailed balance. 


\section{B. Entropy flux and local detailed balance}

The Einstein relation, also called the second fluctuationdissipation relation, connects the noise correlations to the memory kernel in the friction. For an equilibrium dynamics, say taking $F=h \equiv 0$ in (1), that relation [which will follow as (10) below] is most simply derived from the requirement that the stationary velocity distribution should be Maxwellian at inverse temperature $\beta$. For our nonequilibrium dynamics (nonconservative forcing $F$ ), we have in general no information about that stationary distribution. Nevertheless, more fundamentally that Einstein relation arises in the weak coupling limit for the dynamical degrees of freedom with a large thermal bath as a consequence of the microscopic reversibility of the bath degrees of freedom. For our dynamics we do keep the bath in thermal equilibrium and our driven particle does not react back on the bath. That is the basic reason why we will be able to maintain the standard Einstein relation. As the situation with memory is formally more complicated and since we do not want to recall the details of a weak coupling analysis, we can rely on the so-called condition of local detailed balance (see also [4]). It amounts to assuming that, if the system were subjected to a confining force, it would reach equilibrium, and that the exchanges with the thermostat are the same as when the exerted forces drive it out of equilibrium. Formally, this means that the physical entropy flux can be recovered from the time-antisymmetric part of the action. That principle is indeed rooted in the reversibility of the underlying microscopic dynamics as shown in [11]. To be more specific about time reversal we introduce the time-reversal operator $\theta$ according to which

$$
\begin{aligned}
& \theta x_{t}=x_{-t}, \quad \theta v_{t}=-v_{-t}, \\
& \theta h_{t}=h_{-t}, \quad \theta F_{t}=F_{-t},
\end{aligned}
$$

where the last line also includes the time reversal of the protocol (the time dependence in the nonmagnetic external forces). When the $\eta_{t}=\left(\eta_{t}^{i}\right)$ would be multidimensional, we should also assume that the noise is time-reversal invariant in the sense that $\left\langle\eta_{t}^{i} \eta_{0}^{j}\right\rangle=\left\langle\eta_{t}^{j} \eta_{0}^{i}\right\rangle$. Demanding that the system obeys local detailed balance then means to require that the time-antisymmetric part of the action for $\mathcal{P}_{0}$ [first appearing in (4)] is given by the entropy flux in the original (unperturbed) model; that is to say,

$$
\log \frac{d \mathcal{P}_{0}}{d \mathcal{P}_{0} \theta}(\omega)=-\beta \int d s \dot{v}_{s} v_{s}+\beta \int d s F_{s}\left(x_{s}\right) v_{s} .
$$

The first term in the right-hand side is a temporal boundary term accounting for the kinetic energy difference between the initial and final states of the trajectory.

As is explicitly shown in Appendix A, local detailed balance (7) is verified whenever

$$
\left\langle\eta_{s} \eta_{t}\right\rangle=\frac{1}{2}[\gamma(t-s)+\gamma(s-t)]=\frac{1}{2} \gamma(|t-s|)
$$

between the noise covariance and the symmetric part of the memory kernel. We repeat that (8) is as such independent of $F$ (nonequilibrium driving) or $h$ (perturbation) as it expresses the thermal equilibrium of the bath; it formally appears [as (7)] from requiring that the source of time-reversal breaking equals the (excess) entropy flux. The very same condition (8) then also ensures that

$$
\mathcal{A}_{h}(\theta \omega)-\mathcal{A}_{h}(\omega)=\beta \int d s v_{s} h_{s}
$$

with the right-hand side equal to the path-dependent excess entropy flux towards the environment at inverse temperature $\beta$, or the dissipated power by the force $h_{t}$ (setting $k_{B}=1$ ). The identity (9) is also explicitly discussed in Appendix A: we can derive (8) from requiring that the time-antisymmetric part of the action $\mathcal{A}_{h}$ is the excess entropy flux caused by the force $h_{t}$, thus equal to $\beta \int d s v_{s} h_{s}$. We will use the notation $\mathcal{S}^{e x}(\omega)=\mathcal{A}_{h}(\theta \omega)-\mathcal{A}_{h}(\omega)$ for (9) in what follows. It is worth to note that mathematically (8) leads to simply rewriting (2) as

$$
\int d s \Gamma(t-s) \gamma(|s|)=2 \delta(t)
$$

\section{The time-symmetric part, or the activity}

The time-symmetric part of the action $\mathcal{A}_{h}$ is $\mathcal{T}^{e x}(\omega)=$ $\mathcal{A}_{h}(\theta \omega)+\mathcal{A}_{h}(\omega)$ and is calculated to be

$$
\begin{aligned}
\mathcal{T}^{e x}(\omega)= & \beta \int d r H_{r}\left[F_{r}\left(x_{r}\right)-\dot{v}_{r}\right] \\
& +\frac{\beta}{2} \int d u v_{u} \int d r H_{r}[\gamma(u-r)-\gamma(r-u)],
\end{aligned}
$$

where we have introduced the "smeared-out" perturbation

$$
H_{r}=\int d s h_{s} \Gamma(r-s)
$$

This time-symmetric part $\mathcal{T}^{e x}$ is a function on path space and is also called the dynamical activity; it is related to the frenetic contribution in linear response playing an important role when away from equilibrium (see [2,3]). Note that the antisymmetric part of the memory kernel vanishes in the Markov case. In this limiting case, $H_{s}=h_{s} / \gamma$ and then

$$
\mathcal{T}_{\text {Markov }}^{\text {ex }}(\omega)=\frac{\beta}{\gamma} \int d s h_{s}\left[F_{s}\left(x_{s}\right)-\dot{v}_{s}\right]
$$

to linear order in $h_{t}$, as before.

\section{LINEAR RESPONSE RELATIONS}

In what follows, we denote by $\langle\ldots\rangle_{h},\langle\ldots\rangle$ the average over the paths generated by (1) with or without the perturbation $h_{t}$. The only randomness over which we average is the stationary noise $\eta_{t}$, but sometimes an additional average over initial conditions will be mentioned. For a general observable local in time we write $O\left(x_{t}, v_{t}\right)=O_{t}$.

\section{A. General susceptibility}

The linear response of observable $O$ is obtained from

$$
\left\langle O_{t}\right\rangle_{h}-\left\langle O_{t}\right\rangle=-\left\langle O_{t} \mathcal{A}_{h}\right\rangle
$$

or in terms of the generalized susceptibility $\chi_{O}$ defined by

$$
\chi_{O}(s, t)=\left.\frac{\delta}{\delta h_{s}}\left\langle O_{t}\right\rangle_{h}\right|_{h=0} .
$$


From inserting (5) into (12), we find

$$
\begin{aligned}
\chi_{O}(s, t)= & \frac{\beta}{2} \int d r \int d u \Gamma(r-s) \gamma(r-u)\left\langle O_{t} v_{u}\right\rangle \\
& +\frac{\beta}{2} \int d r \Gamma(r-s)\left(\left\langle\dot{v}_{r} O_{t}\right\rangle-\left\langle F_{r}\left(x_{r}\right) O_{t}\right\rangle\right) .
\end{aligned}
$$

There are different ways to write that same formula. We can add and subtract to get the symmetric part of the memory kernel:

$$
\begin{aligned}
\chi_{O}(s, t)= & \beta\left\langle O_{t} v_{s}\right\rangle-\frac{\beta}{2} \int d r \int d u \Gamma(r-s) \gamma(u-r)\left\langle O_{t} v_{u}\right\rangle \\
& +\frac{\beta}{2} \int d r \Gamma(r-s)\left(\left\langle\dot{v}_{r} O_{t}\right\rangle-\left\langle F_{r}\left(x_{r}\right) O_{t}\right\rangle\right)
\end{aligned}
$$

Another possibility is to consider separately the timeantisymmetric and the time-symmetric parts. In that case, we follow the decomposition of the action $\mathcal{A}_{h}=\left(\mathcal{T}^{e x}-\mathcal{S}^{e x}\right) / 2$, and from (12) the susceptibility reads as

$$
\chi_{O}(s, t)=\frac{1}{2}\left\langle\sigma_{s} O_{t}\right\rangle-\frac{1}{2}\left\langle\tau_{s} O_{t}\right\rangle,
$$

where

$$
\sigma_{s}=\left.\frac{\delta}{\delta h_{s}} \mathcal{S}^{e x}\right|_{h=0}=\beta v_{s}
$$

and

$$
\begin{aligned}
\tau_{s}= & \left.\frac{\delta}{\delta h_{s}} \mathcal{T}^{e x}\right|_{h=0}=\beta \int d r \Gamma(r-s)\left(F_{r}-\dot{v}_{r}\right) \\
& +\frac{\beta}{2} \int d u \int d r v_{u} \Gamma(r-s)[\gamma(u-r)-\gamma(r-u)] .
\end{aligned}
$$

The formulation (15) separates an entropic from a frenetic contribution as suggested, e.g., in [3]. The formulas (13), (14), and (15) are the first principal results of the paper; they give a general understanding of the structure of nonequilibrium response also in the presence of memory effects. Moreover, there is the promise in each of the last terms in their right-hand sides to learn about the nonequilibrium driving exactly by the study of the response and especially from the frenetic contribution.

The deviation from the Markov case is felt only in this excess dynamical activity $\mathcal{T}^{e x}$ and not in the excess entropy flux $\mathcal{S}^{e x}$. That explains why only in nonequilibrium situations the fluctuation-response relations change when going from Markov to non-Markov; in equilibrium, only the entropy fluxes enter in fluctuation-response relations. Of course, the transient diffusive case is a nonequilibrium situation, and we should be careful when possibly identifying $F_{r}=0$ with the equilibrium case.

\section{B. Consequence of causality}

Causality requires that observations before a certain time are not influenced by perturbations after that time. As a consequence, from (15),

$$
\left\langle\sigma_{u} O_{r}\right\rangle=\left\langle\tau_{u} O_{r}\right\rangle
$$

for a time ordering $u>r$. But suppose now that the averages satisfy time-reversal invariance so that

$$
\left\langle\tau_{s} O_{t}\right\rangle=\operatorname{sgn} O\left\langle\tau_{-s} O_{-t}\right\rangle, \quad \operatorname{sgn} O\left\langle\sigma_{-s} O_{-t}\right\rangle=-\left\langle\sigma_{s} O_{t}\right\rangle,
$$

where $\operatorname{sgn} O$ is the parity of observable $O$ under time reversal. Then, under that time reversibility and as a result of the causality relation (17) for $u=-s>r=-t$,

$$
\begin{aligned}
\left\langle\sigma_{s} O_{t}\right\rangle-\left\langle\tau_{s} O_{t}\right\rangle & =\left\langle\sigma_{s} O_{t}\right\rangle-\operatorname{sgn} O\left\langle\tau_{-s} O_{-t}\right\rangle \\
& =\left\langle\sigma_{s} O_{t}\right\rangle-\operatorname{sgn} O\left\langle\sigma_{-s} O_{-t}\right\rangle \\
& =\left\langle\sigma_{s} O_{t}\right\rangle+\left\langle\sigma_{s} O_{t}\right\rangle=2 \beta\left\langle v_{s} O_{t}\right\rangle,
\end{aligned}
$$

which, upon inserting in (15), yields the standard fluctuationdissipation relation

$$
\chi_{o}(s, t)=\beta\left\langle O_{t} v_{s}\right\rangle \Theta(t-s) .
$$

The next section comes back to this with yet another derivation.

Another consequence of causality is that the action (5) verifies

$$
\left\langle O_{t} \mathcal{A}_{h}\right\rangle=0
$$

when $h_{s}=0$ for $s \leqslant t$. That immediately implies that for all $s>t$,

$$
\begin{aligned}
& \int d r \int d u \Gamma(r-s) \gamma(r-u)\left\langle O_{t} v_{u}\right\rangle \\
& =\int d r \Gamma(r-s)\left(\left\langle F_{r}\left(x_{r}\right) O_{t}\right\rangle-\left\langle\dot{v}_{r} O_{t}\right\rangle\right) .
\end{aligned}
$$

There is a simpler identity that applies when the original dynamics is time homogeneous, i.e., when $F_{t}=F$ does not explicitly depend on time $t$. Then, we can think of the unperturbed averages $\langle\ldots\rangle$ as a steady regime. In that case, we take time $t$ very negative in (20), multiply both sides with $\left\langle\eta_{s} \eta_{w}\right\rangle$ for arbitrary $w>t$, integrate over all $s$, and use the identity (2) to obtain

$$
\int_{t}^{+\infty} d u \gamma(w-u)\left\langle O_{t} v_{u}\right\rangle=\left\langle F\left(x_{w}\right) O_{t}\right\rangle-\left\langle\dot{v}_{w} O_{t}\right\rangle .
$$

In the Markov case, this identity is, for $w>t$,

$$
\gamma \frac{d}{d w}\left\langle O_{t} x_{w}\right\rangle=\left\langle F\left(x_{w}\right) O_{t}\right\rangle-\left\langle\dot{v}_{w} O_{t}\right\rangle,
$$

which is readily recognized as $\left\langle O_{t} \eta_{w}\right\rangle=0$ for white noise $\eta_{w}, w>t$.

\section{Equilibrium dynamics}

\section{Confined case}

The equilibrium limiting case can be achieved whenever the force field $F$ derives from a potential function. In that case, time-reversal invariance applies, and one should recover from (12) the standard fluctuation-dissipation theorem (19). To check this more explicitly, we first consider the response of an observable $O_{t} \equiv O\left(x_{t}, v_{t}\right)$ which is even under time reversal (i.e., $\theta O_{t}=O_{-t}$ ). In this case,

$$
\begin{aligned}
\left\langle O_{t} v_{-u}\right\rangle & =-\left\langle O_{-t} v_{u}\right\rangle, \\
\left\langle F_{r}\left(x_{r}\right) O_{-t}\right\rangle & =\left\langle F_{-r}\left(x_{-r}\right) O_{t}\right\rangle, \\
\left\langle\dot{v}_{r} O_{-t}\right\rangle & =\left\langle\dot{v}_{-r} O_{t}\right\rangle .
\end{aligned}
$$

In Appendix B, we show that the time antisymmetric part of the response $\chi$ yields

$$
\chi_{O}(s, t)-\chi_{O}(t, s)=\beta\left\langle O_{t} v_{s}\right\rangle .
$$


By causality, this automatically implies the fluctuationdissipation relation (19).

Likewise, when the observable $O_{t}$ is odd under time reversal (i.e., $\theta O_{t}=-O_{-t}$ ), the right-hand sides in Eq. (22) have to be multiplied by -1 , and thence one has to consider the time-symmetric part $\chi_{O}(s, t)+\chi_{O}(t, s)$ in order to indeed recover (19).

\section{Free diffusion}

We next consider the case where $F_{r}=0$ and there is no confinement on the relevant time scales. In this case, the velocity of the particle relaxes alright to a Maxwellian steady state in the long time limit, but its position diffuses. (It could be anomalous diffusion for slowly decaying kernels.) Therefore, starting from a fixed position and Maxwellian velocity, the velocity response $\chi_{v}$ satisfies

$$
\chi_{v}(s, t)=\chi_{v}(0, t-s)=\beta\left\langle v_{s} v_{t}\right\rangle
$$

for $s<t$, but the position dynamics remains in the transient regime. It is, however, possible to recover a formula similar to (19) which involves the mean square displacement $\Delta x^{2}(t)=$ $\left\langle\left(x_{s+t}-x_{s}\right)^{2}\right\rangle$ as we now explain.

We consider the case where the observable $O$ is the position $x$. The position response

$$
\chi_{x}(t)=\left.\frac{\delta}{\delta h}\left\langle x_{t}\right\rangle_{h}\right|_{h=0}=\left.\int_{0}^{t} d s \frac{\delta}{\delta h_{s}}\left\langle x_{t}\right\rangle_{h}\right|_{h=0}
$$

satisfies

$$
\frac{d}{d t} \chi_{x}(t)=\int_{0}^{t} d s \chi_{v}(s, t)=\beta \int_{0}^{t} d s\left\langle v_{s} v_{t}\right\rangle .
$$

On the other hand,

$$
\begin{aligned}
\Delta x^{2}(t) & =\int_{s}^{s+t} d u \int_{s}^{s+t} d r\left\langle v_{u} v_{r}\right\rangle, \\
\frac{d}{d t} \Delta x^{2}(t) & =2 \int_{s}^{s+t} d u\left\langle v_{u} v_{s+t}\right\rangle .
\end{aligned}
$$

As a result, we get the equilibriumlike result

$$
\chi_{x}(t)=\frac{\beta}{2} \frac{d}{d t} \Delta x^{2}(t), \quad t>0
$$

which, for the Markov case, is an identity attributed to Virasoro in [28]. The fact that this relation remains satisfied also in the presence of strong memory is directly caused by $F=0$ and that only the entropic contribution matters.

\section{Modified Sutherland-Einstein relation}

We now show how to connect the mobility of the particle (which is related to the velocity response to a constant force), and the diffusion properties, related to the time behavior of the mean squared displacement. Remember that the perturbing field is a step function $h_{t}=h \Theta(t)$, with constant field $h$. The time-dependent mobility is then defined as

$$
M(t)=\left.\frac{1}{t} \frac{\partial}{\partial h}\left\langle\left(x_{t}-x_{0}\right)\right\rangle_{h}\right|_{h=0}
$$

or

$$
M(t)=\frac{1}{t} \int_{0}^{t} d s \int_{0}^{t} d r \chi_{v}(s, r) .
$$

The time-dependent diffusion coefficient is defined as

$$
\tilde{D}(t)=\frac{1}{2 t} \Delta x^{2}(t)=\frac{1}{2 t} \int_{0}^{t} d r \int_{0}^{t} d s\left\langle v_{s} v_{r}\right\rangle .
$$

From the general linear response (15) we know that

$$
\left\langle v_{s} v_{t}\right\rangle=\frac{2}{\beta} \chi_{v}(s, t)+\frac{1}{\beta}\left\langle\tau_{s} v_{t}\right\rangle,
$$

which can be replaced in (28) and (29) to give a relation between $M, \tilde{D}$, and $\tau$ :

$$
M(t)=\beta \widetilde{D}(t)-\frac{1}{2 t} \int_{0}^{t} d r \int_{0}^{t} d s\left\langle\tau_{s} v_{r}\right\rangle .
$$

We see how the violation of the Sutherland-Einstein relation $M=\beta \tilde{D}$ is related to the time-averaged correlation between displacement and dynamical activity

$$
\begin{aligned}
M(t) & =\beta \widetilde{D}(t)-\frac{1}{2 t} \int_{0}^{t} d s\left\langle\tau_{s}\left(x_{t}-x_{0}\right)\right\rangle \\
& =\beta \widetilde{D}(t)-\frac{1}{2}\left\langle\left(x_{t}-x_{0}\right) \frac{1}{t} \int_{0}^{t} d s \tau_{s}\right\rangle .
\end{aligned}
$$

This formula has a general validity, independent of initial conditions. The time-averaged dynamical activity due to the perturbation has become here an important observable for describing the deviation from the standard Sutherland-Einstein relation. Since $\tau_{s}$ is time symmetric, the last correction term will vanish when time-reversal symmetry gets established; that happens in the case of free diffusion upon averaging over the initial equilibrium distribution for velocities (as in the previous section). In general, however, we can further detail the expression by using the explicit form of $\tau_{s}$ from (16), yielding three terms in the correction:

$$
M(t)=\beta \widetilde{D}(t)+\widetilde{C}_{1}(t)+\widetilde{C}_{2}(t)+\widetilde{C}_{3}(t) .
$$

As we are, however, most interested in the nonequilibrium situation with possible drift, it is useful to discard this effect by considering a slightly different definition of the diffusion coefficient, relabeled here as $D$ :

$$
D(t)=\frac{1}{2 t}\left\langle\left(x_{t}-x_{0}\right) ;\left(x_{t}-x_{0}\right)\right\rangle .
$$

The notation $\langle A, B\rangle$ refers to the connected (also called truncated) correlation function $\langle A B\rangle-\langle A\rangle\langle B\rangle$. The analog of the modified Sutherland-Einstein relation (32) is explicitly 
given by

$$
\begin{aligned}
M(t)= & \beta D(t)+\underbrace{\frac{\beta}{2 t} \int_{0}^{t} d s \int d r \Gamma(r-s)\left\langle\left(x_{t}-x_{0}\right) ; v_{r}\right\rangle}_{C_{1}(t)} \\
& +\underbrace{\frac{\beta}{4 t} \int_{0}^{t} d s\left[\int d r \int d u \Gamma(r-s)\{\gamma(r-u)-\gamma(u-r)\}\left\langle\left(x_{t}-x_{0}\right) ; v_{u}\right\rangle\right]}_{C_{2}(t)} \underbrace{-\frac{\beta}{2 t} \int_{0}^{t} \int d r \Gamma(r-s)\left\langle F_{r}\left(x_{r}\right) ;\left(x_{t}-x_{0}\right)\right\rangle d s}_{C_{3}(t)} .
\end{aligned}
$$

This is the third key relation of this paper [after the general (13)-(15) and the equilibriumlike (26)]. We have postponed a detailed derivation of this result to Appendix C. Notice that the functions $\widetilde{C}_{i}$ of Eq. (32) are simply given by the functions $C_{i}$ with a standard correlation function instead of a connected one. More concretely, if $C_{i}=\langle A, B\rangle$, then $\widetilde{C}_{i}=\langle A B\rangle$. We shall further explore this relation numerically in the next section. The terms $C_{1}$ and $C_{3}$ are also present (although without convolution with $\Gamma$ ) in the Markov case and account for nonequilibrium effects due, respectively, to the inertia and to the nonequilibrium forcing. The term $C_{1}$ vanishes outside the inertial regime. The term $C_{3}$ is most important for the inverse problem of reconstructing the nonequilibrium driving from violation of the Sutherland-Einstein relation. The term $C_{2}$ has no analog in the Markovian case, and is hence only due to memory effects under nonequilibrium dynamics.

\section{E. Examples}

We present here simulation results for the dynamics (1) for some three choices of the driving $F$. The method to generate colored noise is outlined in Appendix D. For the evaluation of the terms in (34), we note that $\Gamma$ appears as a convolution and the Fourier transform of $\Gamma$ is computed from its definition (2).

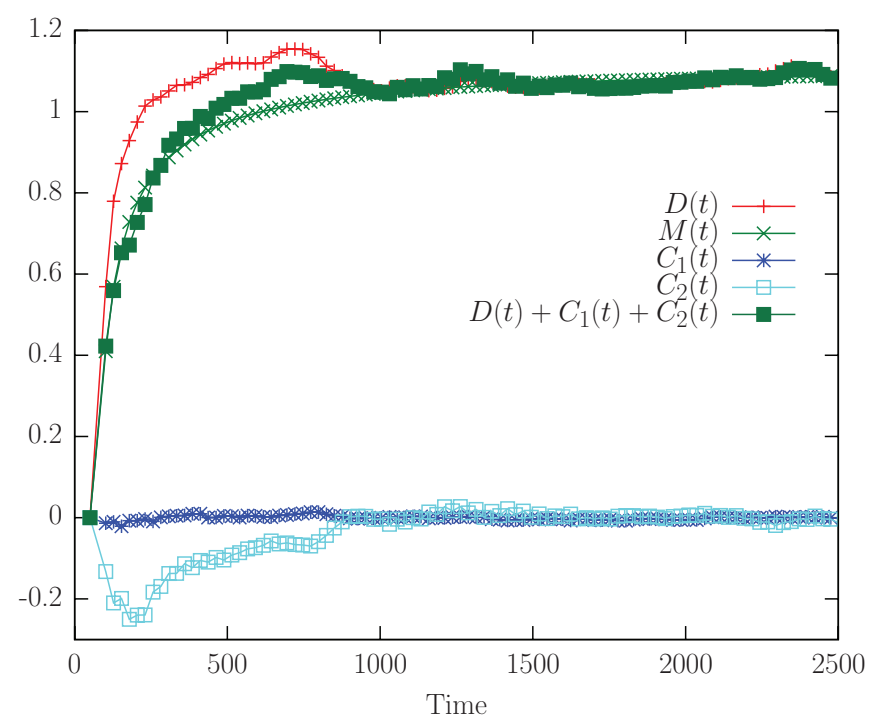

FIG. 1. (Color online) The time dependence of the mobility $M$, the diffusion $D$, and the corrections $C_{1}$ and $C_{2}$ of (34) after multiplying all with $\ln t$. Here, we have long memory $\gamma(t)=\frac{1}{1+t}$ and free diffusion $F=0$ at $\beta=1$.
The purpose is to visualize the various terms in the modified Sutherland-Einstein relation (34), similarly to the work in [1] but with the extra ingredient of memory. Of course, there is no strong need to verify or to confirm the mathematical formula (34) as the linear expansion is sufficiently controlled, but it is somewhat interesting to see the contributions of the various terms in (34) and to see what influences them.

We start with the free diffusion $(F=0)$. The system is diffusive if, for large times, $D(t)$ reaches a limit (the diffusion constant) $D$. On the other hand, when the memory gets long time tails, with $\gamma(t)$ decaying algebraically, the diffusion can become anomalous [29]. We take two different examples, $\gamma(t)=1 /(1+t)$ and $\gamma(t)=1 / \sqrt{1+t}$. As results show, both the diffusion and the mobility follow a corresponding temporal behavior with for the first example $D(t) \simeq 1 / \ln t$ and for the second example $D(t) \simeq 1 / \sqrt{t}$ (subdiffusive motion). The plots in Figs. 1 and 2 show the subdiffusive behavior due to the memory effect. More generally, for free diffusion $D \propto\left[\int_{0}^{t} \gamma(s) d s\right]^{-1}$ for large $t$. Note that we treated free diffusion with some fixed initial condition (without initial velocity averaging) so that the standard Sutherland-Einstein relation gets established only in the long time limit. Note that the inertial regime is rather short lived, $C_{1}$ rapidly being very small, but the memory effect as present in $C_{2}$ postpones the

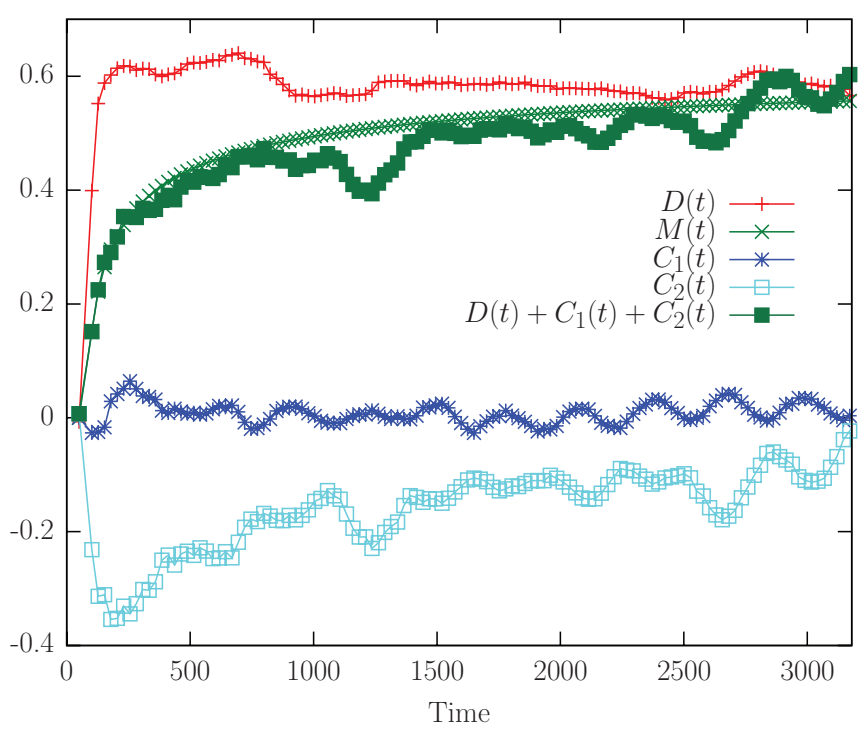

FIG. 2. (Color online) Same case of free diffusion as in Fig. 1 but with a time rescaling of $\sqrt{t}$ for memory kernel $\gamma(t)=\frac{1}{\sqrt{1+t}}$. Still, $F=0, \beta=1$. 


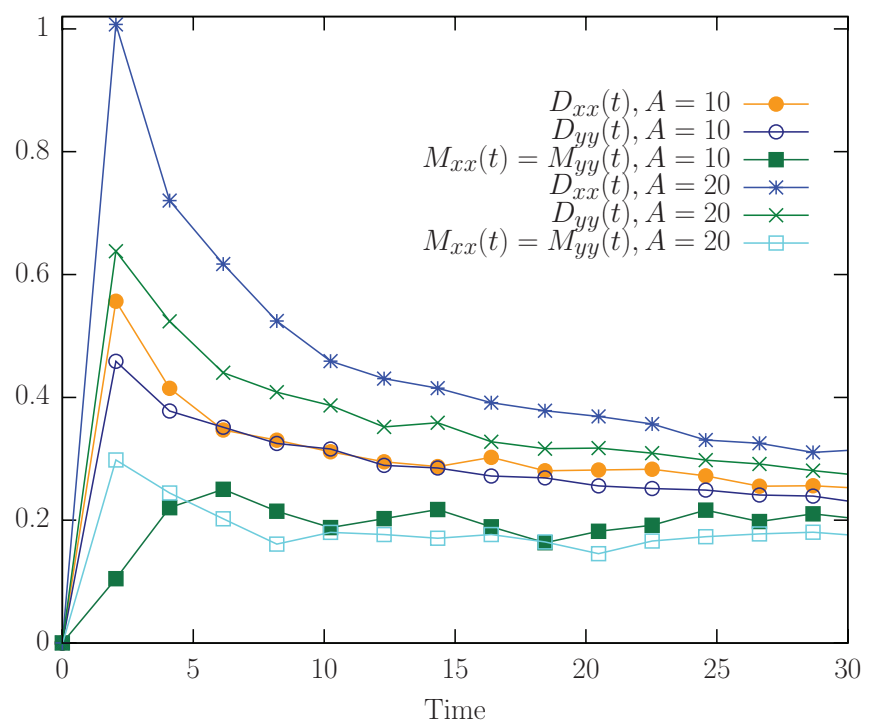

FIG. 3. (Color online) Diffusion and mobility in the case of a two-dimensional rotational forcing (35) for memory $\gamma(t)=\frac{1}{1+t}$ and amplitudes $A=10$ and 20 .

equality between $\beta D(t)$ and $M(t)$ to longer times. The sign of $C_{2}$ carries no special information.

For the other examples, we switched on some rotational force $F$. Again, we checked in all cases that for exponentially decaying memory kernels $\gamma(t)$ the results of [1] are reproduced. We concentrate on power law decay and we consider finite times. In Fig. 3, we consider a vector force on the plane to induce vortices, similar to the rotational force in [1]. We take $\vec{F}=A \vec{g}$, with $A$ the amplitude, where

$$
\begin{aligned}
g_{x}(x, y) & =a(r-\sqrt{2})\left(y-\frac{1}{2}\right), \\
g_{y}(x, y) & =a(r-\sqrt{2})\left(\frac{1}{2}-x\right), \\
a & =\left(1-2 \delta_{2, x \bmod 3}\right)\left(1-2 \delta_{1, y \bmod 2}\right)
\end{aligned}
$$

for distance $r=\sqrt{\left(x-\frac{1}{2}\right)^{2}+\left(y-\frac{1}{2}\right)^{2}}$. The somewhat involved definitions assure that the particle does not undergo a net drift; the forces are purely rotational and not translational now. To make sure, mobility and diffusion are now matrices but the off-diagonal elements are approximately zero. We also find that the diffusion in the $x$ direction is bigger than in the $y$ direction. Moreover, for bigger $A$, the diffusion increases, while the mobility remains almost constant (and even somewhat decreases).

Finally, we consider the result of driving in one dimension. The nonequilibrium force is obtained from a periodic potential, which is like confining the particle to a toroidal trap, and adding a constant field. In the formulas, the nonequilibrium force is $F(x)=A+\sin x$, where $A$ is a constant. Figures 4 and 5 show the result again for the two long-memory kernels. As we have found in the previous section, the relation between diffusion and mobility gets modified. Our simulations confirm in all cases that the diffusion depends on the external forcing more strongly than does the mobility.

In the power law decaying memory for $A=1.5$, the increase of the diffusion is seen. $C_{1}$ is still almost zero, $C_{2}$ vanishes in the long time, and the diffusion and mobility are not

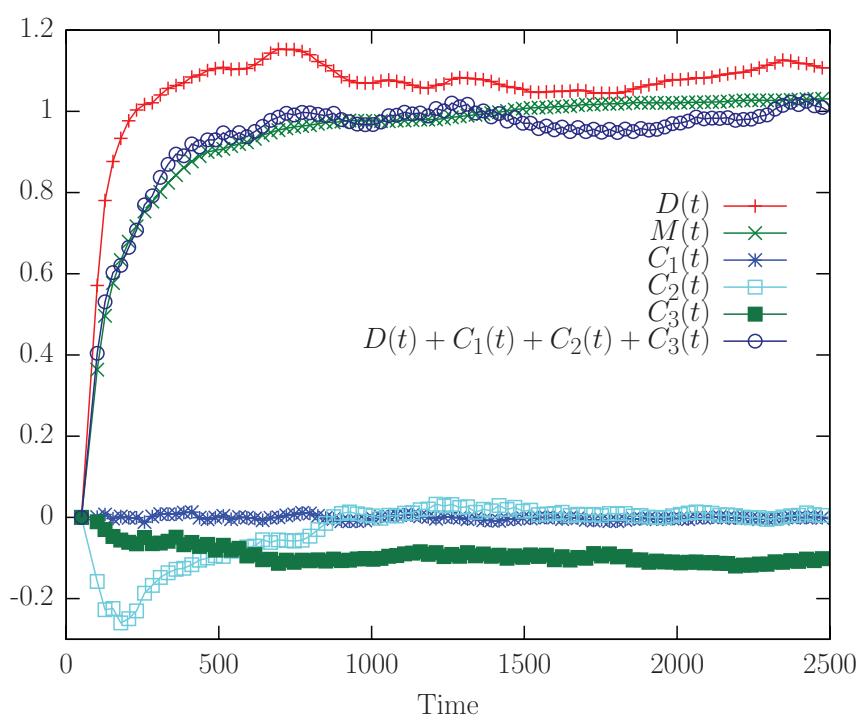

FIG. 4. (Color online) Same setup as in Fig. 1 but for a forcing $F(x)=1.5+\sin x$ (external field over periodic potential) at inverse temperature $\beta=1$. The rescaling of the mobility, the diffusion, and the corrections in (34) is by multiplying all with $\ln t$ for memory kernel $\gamma(t)=\frac{1}{1+t}$.

proportional any more; there is now also the essential term $C_{3}$, which is negative because of the positive correlation between force and displacement. However, for stronger memory and with $A=1.5$ there is little difference with the case $f=0$. To make the contribution of $C_{3}$ more prominent, we have taken a larger driving force, such as $F(x)=8+8 \sin x$ as in Fig. 6; we see that $C_{1}$ is still zero, $C_{2}$ is getting smaller faster than before, and $C_{3}$ is more important now.

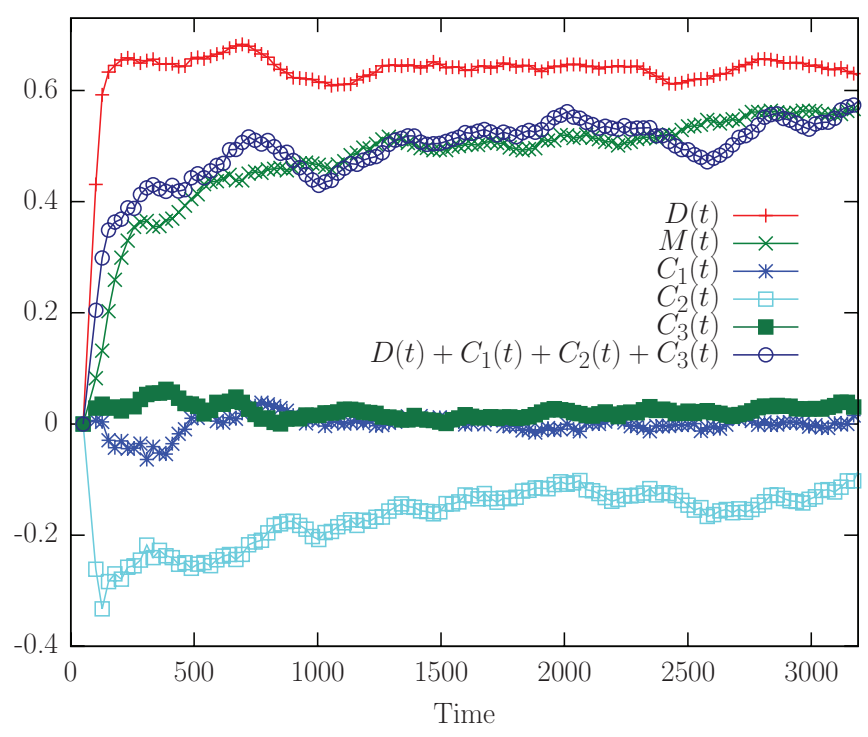

FIG. 5. (Color online) Same setup as in Fig. 2 but for a forcing $F(x)=1.5+\sin x$ (external field over periodic potential) at inverse temperature $\beta=1$. The rescaling of the mobility, the diffusion, and the corrections in (34) is by multiplying all with $\sqrt{t}$ for memory kernel $\gamma(t)=\frac{1}{\sqrt{1+t}}$. 


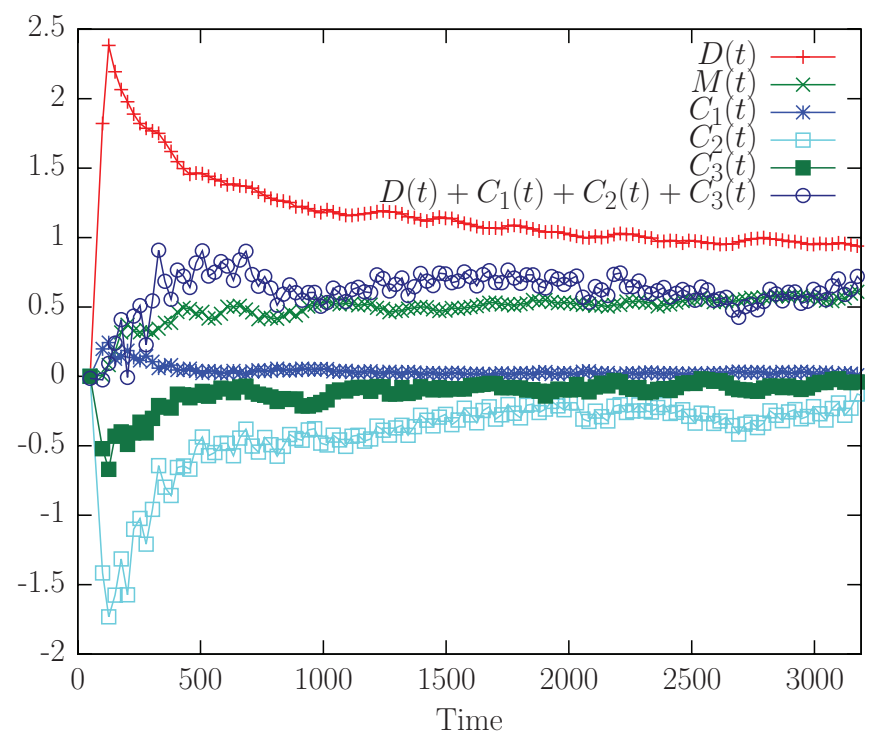

FIG. 6. (Color online) The rescaling of the mobility, diffusion, and the corrections by multiplying with $\sqrt{t}$ for $\gamma(t)=\frac{1}{\sqrt{1+t}}$, and $F(x)=8+8 \sin x, \beta=1$.

\section{RELEVANCE AND CONCLUSION}

The fluctuation-dissipation theorem has an extension to nonequilibrium generalized Langevin systems which preserves the splitting of the response in an entropic and a frenetic contribution. That general statement is of course relevant in today's search for useful linear response formula away from equilibrium. The presence of memory is especially relevant for dense colloidal suspensions. We have here considered a driving which can be time inhomogeneous or even random, but we have also assumed the presence of Gaussian (correlated) noise to start an expansion from path integrals for an underdamped dynamics. The Gaussian correlations are in fact connected with the memory kernel in the friction via the condition of local detailed balance. An important application is to the extension of the Sutherland-Einstein relation between diffusion and mobility. Because of the nonequilibrium condition, the diffusion constant is no longer alone in determining the transport properties of colloidal particles. That was in particular seen in detailed simulations for various nonequilibrium diffusions, possibly anomalous, in particular for exploring the role of the nonequilibrium forcing and the influence of memory. An interesting conclusion is that the nonequilibrium corrections to the Sutherland-Einstein relation are related to the time correlations between the so-called dynamical activity and the velocity of the particle, which in turn leads to information about the correlations between the driving force and the particle's displacement [7]. Such an analysis provides a more general framework for discussions on the violation of the fluctuation-dissipation theorem and is an alternative for the use of effective temperatures. We believe that both the direct question (predicting the response) as the inverse question (deriving information on the nonequilibirum forcing) can be attacked within the given formalism.

\section{ACKNOWLEDGMENTS}

C.M. is grateful to Matthias Krüger for interesting discussions and acknowledges the hospitality in the Condensed Matter Theory group at MIT.

\section{APPENDIX A: CALCULATION OF THE ENTROPY FLUXES}

We start with deriving (7). From writing out the action $\log \frac{d \mathcal{P}_{0}}{d \mathcal{P}_{0} \theta}(\omega)$ in the same way as for (5), we find that

$$
\begin{aligned}
\log \frac{d \mathcal{P}_{0}}{d \mathcal{P}_{0} \theta}(\omega)= & -\frac{\beta}{2} \int d s \int d r \Gamma(r-s) \dot{v}_{r} \int d u[\gamma(r-u)+\gamma(u-r)] v_{u}-\frac{\beta}{4} \int d s \int d r \Gamma(r-s) \int d u \gamma(s-u) v_{u} \\
& \times \int d w \gamma(r-w) v_{w}+\frac{\beta}{4} \int d s \int d r \Gamma(r-s) \int d u \gamma(u-s) v_{u} \int d w \gamma(w-r) v_{w} \\
& +\frac{\beta}{2} \int d s \int d r \Gamma(r-s) F_{r}\left(x_{r}\right) \int d u[\gamma(r-u)+\gamma(u-r)] v_{u}
\end{aligned}
$$

which can be rewritten as

$$
\begin{aligned}
= & -\frac{\beta}{2} \int d s \int d r \Gamma(r-s) \dot{v}_{r} \int d u[\gamma(r-u)+\gamma(u-r)] v_{u}-\frac{\beta}{4} \int d s \int d r \Gamma(r-s) \int d u \gamma(s-u) v_{u} \int d w \gamma(r-w) v_{w} \\
& -\frac{\beta}{4} \int d s \int d r \Gamma(r-s) \int d u \gamma(u-s) v_{u} \int d w \gamma(r-w) v_{w}+\frac{\beta}{4} \int d s \int d r \Gamma(r-s) \int d u \gamma(u-s) v_{u} \int d w \gamma(r-w) v_{w} \\
& +\frac{\beta}{4} \int d s \int d r \Gamma(r-s) \int d u \gamma(u-s) v_{u} \int d w \gamma(w-r) v_{w}+\frac{\beta}{2} \int d s \int d r \Gamma(r-s) F_{r}\left(x_{r}\right) \int d u[\gamma(r-u)+\gamma(u-r)] v_{u}
\end{aligned}
$$


from which we arrive at

$$
\begin{aligned}
\log \frac{d \mathcal{P}_{0}}{d \mathcal{P}_{0} \theta}(\omega)= & -\frac{\beta}{2} \int d s \int d r \Gamma(r-s) \dot{v}_{r} \int d u[\gamma(r-u)+\gamma(u-r)] v_{u}-\frac{\beta}{4} \int d s \int d r \Gamma(r-s) \int d u \int d w[\gamma(s-u) \\
& +\gamma(u-s)] \gamma(r-w) v_{w} v_{u}+\frac{\beta}{4} \int d s \int d r \Gamma(r-s) \int d u \int d w[\gamma(r-w)+\gamma(w-r)] \gamma(u-s) v_{w} v_{u} \\
& +\frac{\beta}{2} \int d s \int d r \Gamma(r-s) F_{r}\left(x_{r}\right) \int d u[\gamma(r-u)+\gamma(u-r)] v_{u} .
\end{aligned}
$$

Since $\left\langle\eta_{s} \eta_{t}\right\rangle=\frac{1}{2}[\gamma(t-s)+\gamma(s-t)]$, as implied by local detailed balance (8), and by using the definition of the symmetric kernel $\Gamma(t)$, the second and the third terms of the right-hand side cancel each other and one finally recovers (7).

Likewise, when we add a time-dependent perturbation $h_{t}$ we have

$$
\begin{aligned}
\log \frac{d \mathcal{P}_{h}}{d \mathcal{P}_{h} \theta}(\omega)= & -\frac{\beta}{2} \int d s \int d r \Gamma(r-s) \dot{v}_{r} \int d u[\gamma(r-u)+\gamma(u-r)] v_{u}-\frac{\beta}{4} \int d s \int d r \Gamma(r-s) \int d u \int d w[\gamma(s-u) \\
& \times \gamma(r-w)-\gamma(u-s) \gamma(w-r)] v_{u} v_{w}+\frac{\beta}{2} \int d s \int d r \Gamma(r-s) F_{r}\left(x_{r}\right) \int d u[\gamma(r-u)+\gamma(u-r)] v_{u} \\
& +\frac{\beta}{2} \int d s \int d r \Gamma(r-s) h_{s} \int d w[\gamma(w-r)+\gamma(r-w)] v_{w} .
\end{aligned}
$$

The excess entropy flux is then

$$
\mathcal{S}^{e x}(\omega)=\mathcal{A}_{h}(\theta \omega)-\mathcal{A}_{h}(\omega)=\log \frac{d \mathcal{P}_{h}}{d \mathcal{P}_{h} \theta}(\omega)-\log \frac{d \mathcal{P}_{0}}{d \mathcal{P}_{0} \theta}(\omega)=\frac{\beta}{2} \int d s \int d r \Gamma(r-s) h_{s} \int d w[\gamma(w-r)+\gamma(r-w)] v_{w}
$$

which indeed ensures (9) upon using (10).

\section{APPENDIX B: RECOVERY OF THE EQUILIBRIUM FLUCTUATION-DISSIPATION THEOREM}

We show how to get from (12) to (23) when (22) holds. We start by writing

$$
\begin{aligned}
\chi_{O}(s, t)-\chi_{O}(t, s)= & \frac{\beta}{2} \int d r \int d u \Gamma(r-s) \gamma(r-u)\left\langle O_{t} v_{u}\right\rangle+\frac{\beta}{2} \int d r \Gamma(r-s)\left(\left\langle\dot{v}_{r} O_{t}\right\rangle-\left\langle F_{r} O_{t}\right\rangle\right) \\
& -\frac{\beta}{2} \int d r \int d u \Gamma(r-t) \gamma(r-u)\left\langle O_{s} v_{u}\right\rangle-\frac{\beta}{2} \int d r \Gamma(r-t)\left(\left\langle\dot{v}_{r} O_{s}\right\rangle-\left\langle F_{r} O_{s}\right\rangle\right) .
\end{aligned}
$$

In the last two integrals, we can perform the change of variable $r^{\prime}=t+s-r$ and $u^{\prime}=t+s-u$. By relabeling $r^{\prime}=r$ and $u^{\prime}=u$, one gets

$$
\begin{aligned}
\chi_{O}(s, t)-\chi_{O}(t, s)= & \frac{\beta}{2} \int d r \int d u \Gamma(r-s) \gamma(r-u)\left\langle O_{t} v_{u}\right\rangle-\frac{\beta}{2} \int d r \int d u \Gamma(r-s) \gamma(u-r)\left\langle O_{s} v_{t+s-u}\right\rangle \\
& +\frac{\beta}{2} \int d r \Gamma(r-s)\left[\left\langle\dot{v}_{r} O_{t}\right\rangle-\left\langle F_{r} O_{t}\right\rangle-\left\langle\dot{v}_{t+s-r} O_{s}\right\rangle+\left\langle F_{t+s-r} O_{s}\right\rangle\right]
\end{aligned}
$$

Finally, by using the time-translation invariance of correlation functions plus the time-reversal conditions (22), one will observe that the square bracket terms in the last integral vanish and the first two integrals simplify thanks to (8) and yield to Eq. (23).

\section{APPENDIX C: MODIFIED SUTHERLAND-EINSTEIN RELATION IN CONNECTED FORM}

By inserting the dynamical activity in (31), we obtain

$$
\begin{aligned}
M(t)= & \frac{\beta}{2 t}\left\langle\left(x_{t}-x_{0}\right)^{2}\right\rangle+\frac{\beta}{2 t} \int_{0}^{t} d s \int d r \Gamma(r-s)\left\langle\left(x_{t}-x_{0}\right) v_{r}\right\rangle+\frac{\beta}{4 t} \int_{0}^{t} d s \int d r \int d u \Gamma(r-s)\{\gamma(r-u) \\
& -\gamma(u-r)\}\left\langle\left(x_{t}-x_{0}\right) v_{u}\right\rangle-\frac{\beta}{2 t} \int_{0}^{t} \int d r \Gamma(r-s)\left\langle F_{r}\left(x_{r}\right)\left(x_{t}-x_{0}\right)\right\rangle d s .
\end{aligned}
$$


We now rewrite this relation as follows:

$$
\begin{aligned}
M(t)= & \frac{\beta}{2 t}\left\langle\left(x_{t}-x_{0}\right)^{2}\right\rangle-\frac{\beta}{2 t}\left\langle x_{t}-x_{0}\right\rangle\left\langle x_{t}-x_{0}\right\rangle+\frac{\beta}{2 t}\left\langle x_{t}-x_{0}\right\rangle\left\langle x_{t}-x_{0}\right\rangle+\frac{\beta}{2 t} \int_{0}^{t} d s \int d r \Gamma(r-s)\left\langle\left(x_{t}-x_{0}\right) \dot{v}_{r}\right\rangle \\
& -\frac{\beta}{2 t} \int_{0}^{t} d s \int d r \Gamma(r-s)\left\langle x_{t}-x_{0}\right\rangle\left\langle\dot{v}_{r}\right\rangle+\frac{\beta}{2 t} \int_{0}^{t} d s \int d r \Gamma(r-s)\left\langle x_{t}-x_{0}\right\rangle\left\langle\dot{v}_{r}\right\rangle \\
& +\frac{\beta}{4 t} \int_{0}^{t} d s \int d r \int d u \Gamma(r-s)\{\gamma(r-u)-\gamma(u-r)\}\left\langle\left(x_{t}-x_{0}\right) v_{u}\right\rangle-\frac{\beta}{4 t} \int_{0}^{t} d s \int d r \int d u \Gamma(r-s)\{\gamma(r-u) \\
& -\gamma(u-r)\}\left\langle x_{t}-x_{0}\right\rangle\left\langle v_{u}\right\rangle+\frac{\beta}{4 t} \int_{0}^{t} d s \int d r \int d u \Gamma(r-s)\{\gamma(r-u)-\gamma(u-r)\}\left\langle x_{t}-x_{0}\right\rangle\left\langle v_{u}\right\rangle \\
& -\frac{\beta}{2 t} \int_{0}^{t} \int d r \Gamma(r-s)\left\langle F_{r}\left(x_{r}\right)\left(x_{t}-x_{0}\right)\right\rangle d s+\frac{\beta}{2 t} \int_{0}^{t} \int d r \Gamma(r-s)\left\langle F_{r}\left(x_{r}\right)\right\rangle\left\langle x_{t}-x_{0}\right\rangle d s \\
& -\frac{\beta}{2 t} \int_{0}^{t} \int d r \Gamma(r-s)\left\langle F_{r}\left(x_{r}\right)\right\rangle\left\langle x_{t}-x_{0}\right\rangle d s .
\end{aligned}
$$

Following the definition of the connected correlation function, we arrive at

$$
\begin{aligned}
M(t)= & \beta D(t)+C_{1}(t)+C_{2}(t)+C_{3}(t)+\frac{\beta}{2 t}\left\langle x_{t}-x_{0}\right\rangle\left\langle x_{t}-x_{0}\right\rangle+\frac{\beta}{2 t} \int_{0}^{t} d s \int d r \Gamma(r-s)\left\langle x_{t}-x_{0}\right\rangle\left\langle\dot{v}_{r}\right\rangle \\
& +\frac{\beta}{4 t} \int_{0}^{t} d s \int d r \int d u \Gamma(r-s)\{\gamma(r-u)-\gamma(u-r)\}\left\langle x_{t}-x_{0}\right\rangle\left\langle v_{u}\right\rangle-\frac{\beta}{2 t} \int_{0}^{t} \int d r \Gamma(r-s)\left\langle F_{r}\left(x_{r}\right)\right\rangle\left\langle x_{t}-x_{0}\right\rangle d s .
\end{aligned}
$$

From the Langevin equation, we have

$$
\left\langle\dot{v}_{r}\right\rangle=-\int d u \gamma(r-u)\left\langle v_{u}\right\rangle+\left\langle F_{r}\left(x_{r}\right)\right\rangle .
$$

After substituting this in (C2), all the terms cancel out each other and only the first line will remain. The cancellation for the forcing term is clear; and the other terms follow as

$$
\begin{aligned}
- & \frac{\beta}{2} \int_{0}^{t} d s \int d r \int d u \Gamma(r-s) \gamma(r-u)\left\langle v_{u}\right\rangle \\
= & -\frac{\beta}{4} \int_{0}^{t} d s \int d r \int d u \Gamma(r-s) \gamma(r-u)\left\langle v_{u}\right\rangle-\frac{\beta}{4} \int_{0}^{t} d s \int d r \int d u \Gamma(r-s) \gamma(r-u)\left\langle v_{u}\right\rangle \\
& -\frac{\beta}{4} \int_{0}^{t} d s \int d r \int d u \Gamma(r-s) \gamma(u-r)\left\langle v_{u}\right\rangle+\frac{\beta}{4} \int_{0}^{t} d s \int d r \int d u \Gamma(r-s) \gamma(u-r)\left\langle v_{u}\right\rangle \\
= & -\frac{\beta}{4 t} \int_{0}^{t} d s \int d r \int d u \Gamma(r-s)\{\gamma(r-u)+\gamma(u-r)\}\left\langle v_{u}\right\rangle-\frac{\beta}{4 t} \int_{0}^{t} d s \int d r \int d u \Gamma(r-s)\{\gamma(r-u)-\gamma(u-r)\}\left\langle v_{u}\right\rangle \\
= & -\frac{\beta}{2}\left\langle x_{t}-x_{0}\right\rangle-\frac{\beta}{4 t} \int_{0}^{t} d s \int d r \int d u \Gamma(r-s)\{\gamma(r-u)-\gamma(u-r)\}\left\langle v_{u}\right\rangle .
\end{aligned}
$$

\section{APPENDIX D: SIMULATION OF COLORED GAUSSIAN NOISE}

We sketch the algorithm to numerically generate a stationary Gaussian colored noise $\xi_{t}$ for a given time-correlation function $\gamma$. The strategy is to transform to Fourier space as, e.g., in [30,31], then to simulate it there as, e.g., in [32], and then to transform the solution back to real space. We next explain that scheme in more detail.

Suppose the noise satisfies

$$
\langle\xi(t)\rangle=0, \quad\left\langle\xi(t) \xi\left(t^{\prime}\right)\right\rangle=\gamma\left(\left|t-t^{\prime}\right|\right) .
$$

In discrete Fourier space, the noise can be constructed as

$$
\tilde{\xi}\left(\omega_{\mu}\right)=\sqrt{N \tilde{\gamma}\left(\omega_{\mu}\right)} \alpha_{\mu}, \quad \mu=0, \ldots, N-1
$$

with $N$ even, and $\tilde{\xi}\left(\omega_{\mu}\right)$ and $\tilde{\gamma}\left(\omega_{\mu}\right)$ being the Fourier transforms of $\xi(t)$ and $\gamma(t)$, respectively; the $\omega_{\mu}$ is defined as

$$
\omega_{\mu}=2 \pi \frac{\mu-\frac{N}{2}}{N \Delta}, \quad \omega_{N-\mu}=-\omega_{\mu},
$$

where $\Delta$ is the sampling interval of time. Finally, $\alpha_{\mu}$ is a Gaussian complex random number in Fourier space with zero 
mean and correlation [33]

$$
\left\langle\alpha_{\mu} \alpha_{\nu}\right\rangle=\delta_{\mu, N-\nu}, \quad \alpha_{\mu}^{*}=\alpha_{N-\mu}
$$

To generate a Gaussian complex random number with correlation given in (D4), we write $\alpha_{\mu}=a_{\mu}+i b_{\mu}$ in terms of its real and imaginary parts: $\alpha_{\mu}=a_{\mu}+i b_{\mu}$ if $\mu>N / 2$ and otherwise $\alpha_{\mu}=a_{N-\mu}-i b_{N-\mu}$. Here, $a$ and $b$ are two Gaussian real random numbers which are uncorrelated and have zero mean and covariance

$$
\left\langle a_{\mu} a_{\nu}\right\rangle=\frac{1}{2}\left(\delta_{\mu, \nu}+\delta_{\mu, N-v}\right), \quad\left\langle b_{\mu} b_{\nu}\right\rangle=\frac{1}{2}\left(\delta_{\mu, \nu}-\delta_{\mu, N-v}\right) .
$$

It is then straightforward to do the inverse Fourier transform

$$
\xi\left(t_{k}\right)=\frac{1}{N} \sum_{\mu=0}^{N-1} \tilde{\xi}\left(\omega_{\mu}\right) e^{i \omega_{\mu} t_{k}}
$$

and to see that the correlations reproduce (D1).
[1] M. Baiesi, C. Maes, and B. Wynants, Proc. R. Soc. A 467, 2792 (2011).

[2] M. Baiesi and C. Maes, New J. Phys. 15, 013004 (2013).

[3] M. Baiesi, E. Boksenbojm, C. Maes, and B. Wynants, J. Stat. Phys. 139, 492 (2010).

[4] T. Harada and S. I. Sasa, Phys. Rev. Lett. 95, 130602 (2005).

[5] D. Villamaina, A. Baldassarri, A. Puglisi, and A. Vulpiani, J. Stat. Mech. (2009) P07024.

[6] A. Crisanti, A. Puglisi, and D. Villamaina, Phys. Rev. E 85, 061127 (2012).

[7] P. Bohec, F. Gallet, C. Maes, S. Safaverdi, P. Visco, and F. Van Wijland, arXiv:1203.3571.

[8] R. Zwanzig, Phys. Rev. 124, 983 (1961).

[9] H. Mori, Prog. Theor. Phys. 33, 423 (1965).

[10] D. Panja, J. Stat. Mech. (2010) L02001.

[11] C. Maes and K. Netočný, J. Stat. Phys. 110, 269 (2003).

[12] R. Kubo, Rep. Prog. Phys. 29, 255 (1966).

[13] R. Kubo, M. Toda, and N. Hashitsume, Statistical Physics: Nonequilibrium Statistical Mechanics, 2nd ed., Vol. 2 (Springer, Berlin, 1992).

[14] J. M. Brader, M. Siebenbuerger, M. Ballauff, K. Reinheimer, M. Wilhelm, S. J. Frey, F. Weysser, and M. Fuchs, Phys. Rev. E 82, 061401 (2010).

[15] M. Kruger and M. Fuchs, Phys. Rev. E 81, 011408 (2010).

[16] M. Krüger and M. Fuchs, Prog. Theor. Phys. Suppl. 184, 172 (2010).

[17] L. Berthier and J. L. Barrat, J. Chem. Phys. 116, 6228 (2002).
[18] C. Aron, G. Biroli, and L. F. Cugliandolo, J. Stat. Mech. (2010) P11018.

[19] C. Aron, Ph.D. thesis, Université Pierre et Marie Curie, 2010.

[20] J. M. Deutsch and O. Narayan, Phys. Rev. E 74, 026112 (2006).

[21] R. F. Fox, J. Math. Phys. 18, 2331 (1977).

[22] P. Hänggi, Z. Phys. B: Condens. Matter Quanta 31, 407 (1978).

[23] P. Hänggi, Z. Phys. B: Condens. Matter Quanta 75, 275 (1989).

[24] E. A. Novikov, Zh. Eksp. Teor. Fiz. 47, 1919 (1964) [Sov. Phys.-JETP 20, 1290 (1965)].

[25] K. Furutsu, J. Res. Natl. Bur. Stand., Sect. D 67, 303 (1963).

[26] M. D. Donsker, in Analysis in Function Space, edited by W. T. Martin and I. Segal (MIT Press, Cambridge, MA, 1964), pp. 17-30.

[27] M. I. Dykman and I. B. Schwartz, Phys. Rev. E 86, 031145 (2012).

[28] L. F. Cugliandolo, J. Kurchan, and G. Parisi, J. Phys. I (France) 4, 1641 (1994).

[29] N. Pottier, Phys. A (Amsterdam) 390, 2863 (2011).

[30] Kun Lü and Jing-Dong Bao, Phys. Rev. E 72, 067701 (2005).

[31] J. Garca-Ojalvo and J. M. Sancho, Noise in Spatially Extended Systems (Springer, New York, 1999).

[32] W. H. Press, W. T. Vetterling, B. P. Flannery, and S. A. Teukolsky, Numerical Recipes: The Art of Scientific Computing (Cambridge University Press, Cambridge, UK, 2007).

[33] J. M. Porrá, K. G. Wang, and J. Masoliver, Phys. Rev. E 53, 5872 (1996). 